\title{
Glucose Transport Induction
}

National Cancer Institute

\section{Source}

National Cancer Institute. Glucose Transport Induction. NCI Thesaurus. Code C40811.

Glucose Transport Induction consists of the initiation of subcellular or molecular processes directly involved in the physical translocation from one site or compartment to another (typically, extracellular to intracellular) of the simple C6H12O6 monosaccharide, glucose, that acts as an energy source for cells. 